\title{
EEG coherence reflects regional corpus callosum area in Alzheimer's disease
}

\author{
O Pogarell, S J Teipel, G Juckel, L Gootjes, T Möller, K Bürger, G Leinsinger, H-J Möller, U Hegerl, \\ H Hampel
}

Objective: Correlations between corpus callosum size and interhemispheric EEG coherence were investigated as measures of interhemispheric connectivity in patients with Alzheimer's disease.

Methods: 11 patients underwent both magnetic resonance imaging and quantitative electroencephalography to assess corpus callosum size and interhemispheric coherence. For comparison, corpus callosum size was measured in 24 healthy elderly control subjects.

Results: Corpus callosum cross sectional area was significantly reduced in Alzheimer patients relative to controls. Posterior interhemispheric coherence ( $\alpha$ and $\beta$ frequencies) correlated significantly with the size of posterior corpus callosum area, and anterior coherence $(\delta, \theta$, and $\alpha$ frequencies) with the size of anterior corpus callosum area in the Alzheimer patients.

Conclusion: Region specific correlations between corpus callosum size and EEG coherence suggest that the decline in interhemispheric connectivity in Alzheimer's disease results from a specific loss of cortical association neurones projecting through the corpus callosum.

C orpus callosum atrophy has been shown to occur independently of subcortical fibre degeneration in patients with Alzheimer's disease. ${ }^{1-3}$ The commissural fibres of the corpus callosum play a dominant role in the maintenance of interhemispheric connectivity, which can be assessed functionally by EEG coherence analysis, ${ }^{4}$ a measure of similarity between electrical signals of different electrode positions. Coherence analysis indicates the functional connections between underlying cortical regions and is decreased in patients with dementia. ${ }^{5-8}$

In this study, we correlated corpus callosum size with measures of interhemispheric coherence in patients with Alzheimer's disease. A region specific correlation between these two measures would support the hypothesis that corpus callosum atrophy in Alzheimer's disease results from the loss of interhemispheric projecting neurones, leading to a decline in interhemispheric connectivity.

\section{METHODS}

\section{Subjects}

We investigated 11 patients (seven male, four female) with probable Alzheimer's disease according to the NINCDSADRDA criteria.. . Their mean (SD) age was 67 (13) years, and they showed mild (short term memory deficits) to moderately severe (assistance needed with household activities) cognitive decline, with a mean mini-mental state examination (MMSE) score of 17.2 (6.6). All patients had been screened to exclude medical comorbidity according to a standard protocol, ${ }^{2}$ were free of risk factors for cerebrovascular disease, and showed no signs of white matter disease on T2 weighted and FLAIR MRI sequences. They were not on drug treatment at the time of the EEG studies, which followed a drug-free period of at least two weeks.

A group of 24 healthy elderly controls (mean age 62 (9) years, MMSE >28), who participated in the MRI study, was included for comparison of the corpus callosum data with the patient group.

The ethics committee of the University of Munich approved the study, and all subjects or the holders of their Durable Power of Attorney gave their written informed consent.

\section{Magnetic resonance image}

Images were obtained with a $1.5 \mathrm{~T}$ magnetic resonance imaging (MRI) scanner (Magnetom Vision, Siemens, Germany). All subjects underwent a sagittally oriented MPRAGE sequence with 128 partitions and an effective slice thickness of $1.25 \mathrm{~mm}$. We acquired a field of view of $260 \mathrm{~mm}$ with a matrix of $256 \times 256$ pixels, resulting in an in-plane pixel size of $1.0 \times 1.0 \mathrm{~mm}$.

\section{Corpus callosum measurement}

Areas of the corpus callosum and of five callosal subregions were measured in the midsagittal slice of the MPRAGE sequence, as described elsewhere, ${ }^{2}$ using ANALYZE ${ }^{\mathrm{TM}}$ software (Biomedical Imaging Resource, Mayo Foundation, Rochester, Minnesota, USA) on an SGI workstation (Silicon Graphics, Palo Alto, California, USA). Subregions were defined using a simple geometrical construction and were labelled $\mathrm{Cl}$ to $\mathrm{C} 5$ in the rostral-occipital direction. Region $\mathrm{Cl}$ covers the callosal rostrum and genu; regions $\mathrm{C} 2$ to $\mathrm{C} 4$ the anterior, middle, and posterior truncus, respectively; and region $\mathrm{C} 5$, the isthmus and splenium. The number of pixels within each region was summed and multiplied by pixel size to obtain absolute values $\left(\mathrm{mm}^{2}\right)$.

\section{EEG recording and analysis}

An EEG was digitally recorded from 19 electrodes placed according to the 10/20 system, using a computerised EEG unit (Brain Electrical Signal Topography, Austria, 1993) during five minutes with eyes closed and under resting conditions (impedances of electrodes below $5 \mathrm{k} \Omega$, bandpass 0.16 to $70 \mathrm{~Hz}$, sample rate $256 \mathrm{~Hz}$ ). Artefact-free data were processed off-line by bipolar transformation and segmentation into two second epochs. According to the protocol, at least 20 artefact-free segments were required from each subject for fast Fourier transform and power spectral analysis. Interhemispheric coherences were computed

Abbreviations: MMSE, mini-mental state examination; MPRAGE, magnetisation prepared rapid acquisition gradient; NINCDS-ADRDA, National Institute of Neurological and Communicative Disorders and Stroke-Alzheimer's Disease and Related Disorders Association 
between anterior (F3-C3/F4-C4), lateral (F7-T5/F8-T6), and posterior $(\mathrm{C} 3-\mathrm{Ol} / \mathrm{C} 4-\mathrm{O} 2)$ pairs of electrodes, based on the fast Fourier transform for each of the following frequency bands: $\delta(1-3 \mathrm{~Hz}), \theta(4-7 \mathrm{~Hz}), \alpha \mathrm{l}(8-10 \mathrm{~Hz}), \alpha 2(10-12 \mathrm{~Hz})$, $\beta 1(13-18 \mathrm{~Hz}), \beta 2(18-24 \mathrm{~Hz}), \beta 3(24-32 \mathrm{~Hz})$, and total $(1-32$ $\mathrm{Hz}$ ). Coherence is a function of power spectral outputs for the two pairs of electrodes (cross-covariance function) with values between 0 and 1 at a given frequency. A coherence value of 0 indicates random relations between the signals (phases are dispersed); a value of 1 means that the correlated signals are phase locked, indicating shared activity of the channels. Thus coherence is a quantitative measure of the similarity of signals in a given frequency band.

\section{Statistical analysis}

Statistical analyses were carried out using the Statistical Package for the Social Sciences (SPSS Inc, Chicago, Illinois, USA). Corpus callosum areas were compared between Alzheimer patients and controls using analysis of covariance (ANCOVA) with diagnosis as between-subjects factor and age as covariate. The patients' coherences were analysed within the frequency bands for anterior, lateral, and posterior brain regions. Associations between electrophysiological (coherence values) and corpus callosum data were assessed using Spearman's rank correlation coefficients $(\rho)$. In addition, partial correlations were calculated to control for the effects of age and MMSE scores. Probability $(p)$ values below 0.05 were considered statistically significant. Owing to the exploratory character of the study and the small sample size, no $\alpha$ correction was undertaken.

\section{RESULTS}

The mean (SD) cross sectional area of the corpus callosum was $356(65) \mathrm{mm}^{2}$ in the Alzheimer group, which was significantly smaller than the area in the control group (454 (70) $\mathrm{mm}^{2}$ ) as assessed by ANCOVA with the covariate age $(\mathrm{F}=13.2, \mathrm{df}=1,32 ; \mathrm{p}=0.001)$. In the patient group the sizes of the callosal subregions were 98 (25), 43 (11), 41 (8), 39 (12), and $117(23) \mathrm{mm}^{2}$ for $\mathrm{Cl}$ through $\mathrm{C} 5$, respectively. The mean (SD) values of interhemispheric coherences, averaged for the whole frequency spectrum, ranged between 0.21 (0.14) (lateral) and $0.41(0.12)$ (posterior). The frequency distribution revealed the highest interhemispheric coherences for $\alpha$ frequency bands in the posterior regions, and for $\delta$ and $\theta$ frequencies in the frontal regions. The posterior interhemispheric coherences ( $\alpha, \beta$ frequency bands) showed strong positive correlations with the total corpus callosum area $(\rho=+0.76$ and $+0.66, p<0.01$, for $\alpha 2$ and $\beta 1$ coherences, respectively) (table 1 ).

Following the evidence of an anterior-posterior topography of corpus callosum fibre organisation, we correlated corresponding regional coherences and callosal subregions to investigate regional specificity. We found statistically significant correlations between anterior interhemispheric $\delta, \theta$, $\alpha 2$ coherences and callosal subregion $\mathrm{C} 2$, and between

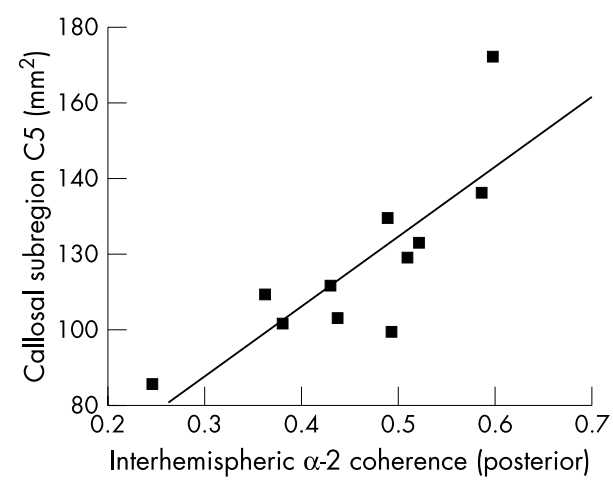

Figure 1 Scatterplot of posterior interhemispheric EEG coherences $(\alpha 2$ frequency band) and posterior corpus callosum sizes (subregion C 5 ) of 11 patients with Alzheimer's disease. Partial correlation (controlled for age, MMSE): $\rho=+0.86, p<0.01$.

posterior $\alpha 2, \beta 1$ coherences and callosal area of subregion C5 (table 1). The posterior correlations remained significant after controlling for age and MMSE $(\rho=+0.86, p<0.01$; fig 1$)$. Inverse analyses - that is, anterior (posterior) coherences and posterior (anterior) corpus callosum subregions-did not show significant correlations (table 1).

\section{DISCUSSION}

We investigated the association between the size of corpus callosum subregions and corresponding regional measures of interhemispheric coherences in patients with Alzheimer's disease. There were strong positive correlations between the total corpus callosum area and interhemispheric posterior $\alpha 2$ and $\beta \mathrm{l}$ coherences. There were also significant positive correlations between anterior/posterior coherences and anterior/posterior callosal subregions. The relevance of the regional distribution of the results was supported by the finding that the inverse analyses showed no significant correlations. The data indicate that corpus callosum area in Alzheimer's disease is directly related to a decrease in functional hemispheric connectivity. The posterior correlations remained significant after controlling for the effects of aging and dementia severity, which suggests that the associations between regional corpus callosum size and interhemispheric connectivity are not merely a consequence of disease progression, but rather reflect a biologically meaningful interaction between the two variables.

The loss of correlations between the size of anterior callosal regions and lower frequency coherences after controlling for age and MMSE is most probably a consequence of the small number of patients under investigation. As the patients showed no sign of subcortical vascular disease on MRI scans, the reduced area of the corpus callosum is likely to reflect a loss of corticocortical projecting neurones, in agreement with previous studies on independent samples showing corpus

Table 1 Correlations of mean regional interhemispheric coherences and corpus callosum sizes in patients with Alzheimer's disease:

\begin{tabular}{llllllll}
\hline Correlations & $\boldsymbol{\delta}$ & $\boldsymbol{\theta}$ & $\boldsymbol{\alpha 1}$ & $\boldsymbol{\alpha 2}$ & $\boldsymbol{\beta 1}$ & $\boldsymbol{\beta 2}$ & $\boldsymbol{\beta 3}$ \\
\hline Posterior coherence-TCA & 0.51 & 0.45 & 0.15 & $\mathbf{0 . 7 6 ^ { * * }}$ & $\mathbf{0 . 6 6 ^ { * * }}$ & 0.34 & 0.22 \\
Posterior coherence-C5 & 0.22 & 0.42 & 0.44 & $\mathbf{0 . 7 7}^{* *}$ & $\mathbf{0 . 5 8}^{*}$ & 0.28 & 0.49 \\
Posterior coherence-C2 & 0.29 & 0.17 & 0.10 & 0.41 & 0.49 & 0.30 & 0.06 \\
Anterior coherence-C2 & $\mathbf{0 . 5 6 ^ { * }}$ & $\mathbf{0 . 5 8 ^ { * }}$ & 0.23 & $\mathbf{0 . 5 6 ^ { * }}$ & 0.46 & 0.36 & 0.13 \\
Anterior coherence-C5 & 0.06 & 0.31 & 0.04 & 0.01 & $\mathbf{0 . 2 7}$ & 0.35 & 0.34 \\
\hline
\end{tabular}

Correlation coefficients $(\rho)$ per EEG frequency band for posterior/anterior coherence and size of total (TCA), anterior (C2), and posterior (C5) corpus callosum area. Significant results in bold: ${ }^{*} \mathrm{p}<0.05,{ }^{* *} \mathrm{p}<0.01$. 
callosum atrophy in the absence of white matter pathology, ${ }^{1-3}$ and significant correlations between corpus callosum atrophy and cortical metabolic decline in Alzheimer's disease. ${ }^{10}{ }^{11}$ In addition, the mean cross sectional corpus callosum area of the patients was significantly lower than in a group of healthy elderly controls, indicating callosal atrophy. Coherence analysis is a measure of alterations in brain connectivity, and previous EEG studies have also revealed lower interhemispheric coherences associated with corpus callosum pathology, ${ }^{12-14}$ providing evidence that interhemispheric coherences are affected by loss or disruption of long axonal connections between the hemispheres.

Unfortunately we were unable to investigate more patients using both structural imaging and electrophysiological techniques, and the small number of subjects clearly compromised the statistical power of this investigation. Furthermore, no correction for multiple comparisons (for example, Bonferroni) was done in view of the exploratory character of the study. Nevertheless, our results show a clear correlation between structural and functional measurements which might well have clinical significance, for example in the milder stages of the disease, especially as the loss of functional connectivity assessed by coherence analysis is one of the most sensitive EEG findings in Alzheimer's disease. ${ }^{56}$

\section{Conclusions}

Our data suggest that atrophy of the corpus callosum in Alzheimer's disease results from cortical neuronal degeneration, leading to a reduction in functionally intact commissural fibres and subsequently to an impairment of brain connectivity. This supports the idea that corpus callosum atrophy contributes to the cortical disconnection syndrome in Alzheimer's disease on a region specific basis.

\section{ACKNOWLEDGEMENTS}

We thank Felician Iancu, Christine Sänger, and Bea Riemenschneider (Ludwig-Maximilians-University of Munich) for technical support. Part of this work was supported by grants from the Medical Faculty of the Ludwig-Maximilians-University, Munich, Germany (SJT), from the Hirnliga eV, Nürmbrecht, Germany (SJT, HH), and from the Competence Network on Dementias funded by the Ministry for Education and Research (BMBF 01 GI 0102), Germany (HH, SJT).

\section{Authors' affiliations \\ O Pogarell, S J Teipel, G Juckel, T Möller, K Bürger, H-J Möller, U Hegerl, H Hampel, Department of Psychiatry, Ludwig-Maximilians- University, Munich, Germany}

G Leinsinger, Department of Radiology, Ludwig-Maximilians-University, Munich

L Gootjes, Department of Clinical Neuropsychology, Free University, Amsterdam, Netherlands

Competing interests: none declared

Correspondence to: Dr Oliver Pogarell, Department of Psychiatry, Ludwig-Maximilians-University of Munich, Nussbaumstrasse 7, D-80336 Munich, Germany; oliver.pogarell@med.uni-muenchen.de

Received 13 January 2004

In revised form 21 April 2004

Accepted 23 April 2004

\section{REFERENCES}

1 Biegon A, Eberling JL, Richardson BC, et al. Human corpus callosum in aging and Alzheimer's disease: a magnetic resonance imaging study. Neurobiol Aging 1994; 15:393-7.

2 Hampel H, Teipel SJ, Alexander GE, et al. Corpus callosum atrophy is a possible indicator of region- and cell type-specific neuronal degeneration in Alzheimer disease: a magnetic resonance imaging analysis. Arch Neurol 1998;55:193-8.

3 Hampel H, Teipel SJ, Alexander GE, et al. Corpus callosum measurement as an in vivo indicator for neocortical neuronal integrity, but not white matter pathology, in Alzheimer's disease. Ann NY Acad Sci 2000;903:470-6.

4 Zaveri HP, Williams WJ, Sackellares JC, et al. Measuring the coherence of intracranial electroencephalograms. Clin Neurophysiol 1999;1 10:1717-25.

5 Leuchter AF, Spar JE, Walter DO, et al. Electroencephalographic spectra and coherence in the diagnosis of Alzheimer's-type and multi-infarct dementia. A pilot study. Arch Gen Psychiatry 1987;44:993-8.

6 Leuchter AF, Newton TF, Cook IA, et al. Changes in brain functional connectivity in Alzheimer-type and multi-infarct dementia. Brain 1992;115:1543-61.

7 Dunkin JJ, Leuchter AF, Newton TF, et al. Reduced EEG coherence in dementia: state or trait marker? Biol Psychiatry 1994;35:870-9.

8 Jelic V, Shigeta $M$, Julin P, et al. Quantitative electroencephalography power and coherence in Alzheimer's disease and mild cognitive impairment. Dementia 1996:7:314-23.

9 McKhann G, Drachman D, Folstein M, et al. Clinical diagnosis of Alzheimer's disease: report of the NINCDS-ADRDA Work Group under the auspices of Department of Health and Human Services Task Force on Alzheimer's Disease. Neurology 1984;34:939-44.

10 Yamauchi H, Fukuyama H, Harada K, et al. Callosal atrophy parallels decreased cortical oxygen metabolism and neuropsychological impairment in Alzheimer's disease. Arch Neurol 1993;50:1070-4.

11 Teipel SJ, Hampel H, Pietrini P, et al. Region-specific corpus callosum atrophy correlates with the regional pattern of cortical glucose metabolism in Alzheimer disease. Arch Neurol 1999;56:467-73.

12 Montplaisir J, Nielsen T, Cote J, et al. Interhemispheric EEG coherence before and after partial callosotomy. Clin Electroencephalogr 1990;21:42-7.

13 Pinkofsky HB, Struve FA, Meyer MA, et al. Decreased multi-band posterior interhemispheric coherence with a lipoma on the corpus callosum: a case report of a possible association. Clin Electroencephalogr 1997;28:155-9.

14 Knyazeva MG, Innocenti GM. EEG coherence studies in the normal brain and after early-onset cortical pathologies. Brain Res Brain Res Rev $2001 ; 36: 119-28$ 